\title{
A behavior field approach to instrumental learning in the rat: II. Training parameters and a stage model of extinction
}

\author{
PAUL T. P. WONG \\ Trent University, Peterborough, Ontario, Canada K9J $7 B 8$
}

\begin{abstract}
Three runway experiments tested a stage model of extinction which postulated an orderly succession of three qualitatively different stages: habit, trial and error, and resolution. The model predicted that Stage 1 should be characterized by perseveration of habitual routes (i.e., response persistence) and the absence of competing responses; Stage 2, by an increase in investigatory behavior (response variation and hole exploration) and biting behavior; Stage 3 , by a decrease in the competing responses of Stage 2 and continued increase in goal avoidance and substitution behavior (e.g., sand-digging). These predictions were largely confirmed. Further, Experiments 1 and 2 showed that, as expected by the model, continuous reinforcement (CRF) resulted in more practice of habitual routes in acquisition and greater response persistence, while partial reinforcement (PRF) resulted in more route variation and hole exploration in acquisition and greater goal persistence which was attributable to prior reinforcement of a trial-anderror coping strategy. Results of Experiment 3, which combined training trials and reward magnitudes orthogonally, supported the prediction that response persistence was positively related to training trials, and goal persistence negatively related to reward magnitudes. All three experiments demonstrated an inverted-U function in investigatory and biting behavior, as predicted by the stage model.
\end{abstract}

A behavioral field may be defined as a matrix of interrelated behaviors in the testing situation. The rationale for a behavioral field approach to instrumental learning has been detailed elsewhere (Wong, 1977). Briefly, this approach favors a molecular analysis of molar behavior-molecular because it advocates multiple recordings and naturalistic observation of various aspects of behavior-environment interactions, and molar because it employs testing situations that permit the occurrence of species-typical behavior and integrated adaptive acts.

Behavioral field analysis of extinction has not been documented in the literature. However, a variety of extinction-induced behavior has been reported, such as aggression (Azrin, Hutchinson, \& Hake, 1966), agitated behavior (Miller \& Stevenson, 1936), escape (Rosellini \& Seligman, 1975), displacement activities (McFarland, 1966), adjunctive drinking (Falk, 1971), sand-digging (Wong, 1977), and response variation (Antonitis, 1951). These phenomena should be taken into account in any viable formulation of experimental extinction.

Supported by NRC Grant A8635 and a University Research Committee grant. The assistance of Helen Orr and Wanda Grife in data collection is gratefully acknowledged. The author is indebted to William Estes and Robert Bolles for their helpful comments on an earlier version of the paper. Requests for reprints should be addressed to Paul T. P. Wong, Department of Psychology, Trent University, Peterborough, Ontario, K9J 7B8, Canada.
To incorporate existing findings on extinctioninduced behavior into frustration theory (Amsel, 1967), a stage model of extinction was developed by the author. According to this model, extinction involves an orderly succession of three qualitatively different stages. In the initial, habit, stage, the predominant coping strategy is perseverationinvigoration, which involves the repetition of a previously reinforced habit at a more vigorous rate. This habitual mode of responding has been described as automatic (Kimble \& Perlmuter, 1970) and ballistic (Bindra, 1969). According to Logan (1971), as well-practiced habit may run off to its completion in the absence of incentive. Similarly, Mandler (1964, p. 169) has stated that once instigated, a habitual response tends to "rush to completion and nothing external to the organism is necessary to insure that continuation of the sequence, even though external factors are crucial for its initiation." Strong evidence for this mode of coping comes from findings of response perseveration resulting in net loss of noncontingent reinforcement (Jensen, 1963; Stolz \& Lott, 1964). Evidence for response invigoration following the initial transition from acquisition to extinction mainly comes from free operant research (Margulies, 1961; Notterman \& Mintz, 1965).

In the second, trial-and-error, stage, the predominant coping strategy may be referred to as variation-aggression. Response variation consists of exploring various members of the operant response 
class and various aspects of the testing situation (McFarland, 1966; Miller \& Stevenson, 1936; Wong, 1977).

In the last, resolution, stage, the initial goal is abandoned in favor of subgoals, such as drinking and sand-digging (Wong, 1977). In the absence of appetitive subgoals, depression may be the consequence (Klinger, 1975; Klinger, Kemble, \& Barta, 1974). The predominant coping strategy in this stage may be described as substitution-depression.

Major features of the stage model are schematized in Figure 1. On a descriptive level, the habit stage is characterized by perseveration of the habitual topography and the absence of competing responses, e.g., exploration and aggression. The onset of the trial-and-error stage is evidenced first by an investigatory behavior (i.e., selection of other response topographies and exploration of the environment) and then an increase in aggression. Later in Stage 2, there should be the emergence of goal avoidance, substitution behavior, and perhaps other displacement activities stemming from approach-avoidance conflict. Finally, transition into the conflict resolution stage is marked by a decrease in the operant, exploratory, and aggressive behavior, but a rapid increase in avoidance and substitution behavior.

The model makes an important distinction between response persistence and goal persistence (Wong, 1977). Response persistence in Stage 1 pertains to the perseveration of a habitual topography; goal persistence in Stage 2 pertains to the pursuit of the initial goal by whatever means. Thus, instead of asking whether a particular acquisition treatment increases resistance to extinction, one asks whether it increases response persistence or goal persistence. The main purpose of the present experiments was to determine how various training parameters differentially affect these two kinds of persistence.

The stage model is essentially an extension of frustration theory. Various behavioral effects of frustrative nonreward have been described (Amsel, 1958; Gray, 1971; Miller, 1959). To integrate these frustration effects, the stage model attempts to specify the temporal sequence as well as the probability distribution of various frustration-induced behaviors in the course of extinction. The present stage analysis is quite consistent with the findings that response invigoration preceded aggression (Thompson \& Bloom, 1966); that response variation preceded discontinuation of the operant (McFarland, 1966; Wong, 1977); that aggressive behavior was an inverted- $U$ function of extinction training (Azrin et al., 1966; Thompson \& Bloom, 1966); and that speed of escape from the unbaited goalbox first increased and then decreased (Rosellini \& Seligman, 1975).

On the theoretical level, anticipatory frustration

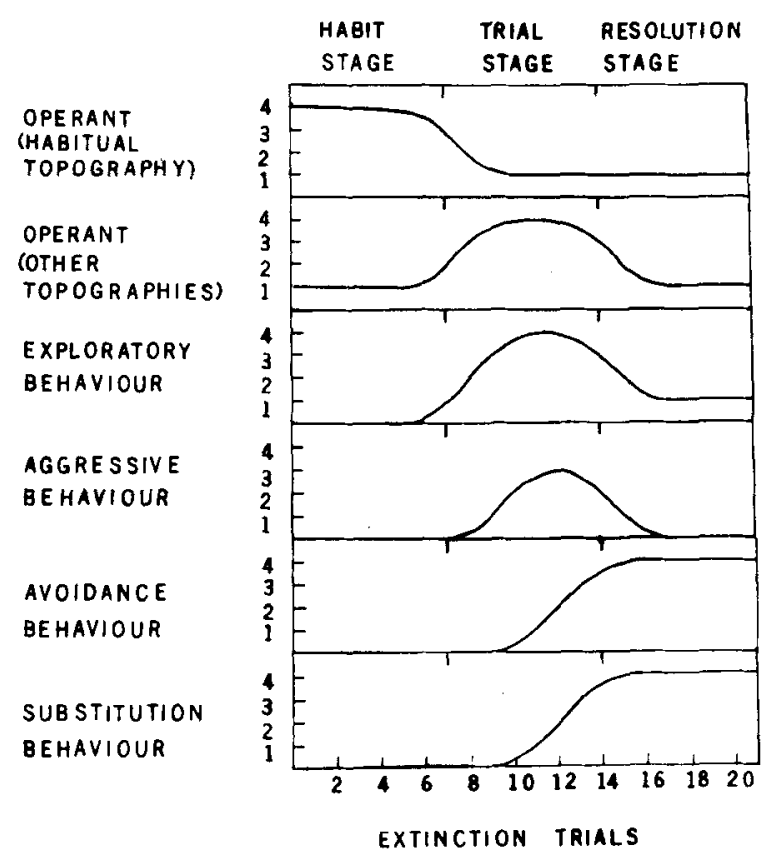

Figure 1. Schema of stage model. The ordinate indicates strength of responding, with four being the maximum and zero the minimum.

$\left(\mathrm{r}_{\mathrm{F}}-\mathrm{S}_{\mathrm{F}}\right)$ is accorded the responsibility for modulating stage transition. In Stage 1, the intensity of $\mathrm{r}_{\mathrm{F}}-\mathrm{S}_{\mathrm{F}}$ is assumed to be too weak to evoke competing responses (cf. Amsel, 1967). As $\mathrm{r}_{F}$ - $_{\mathrm{F}}$ increases in intensity, it mediates investigatory behavior, thus initiating the second stage of extinction. With prolonged nonreward, the intensity of $\mathrm{r}_{\mathrm{F}}-\mathrm{S}_{\mathrm{F}}$ should eventually become so aversive that the tolerance threshold is reached, upon which the approach-avoidance conflict is resolved in favor of alternative goals or substitution behavior. The tolerance of resolution threshold may be determined by such factors as prior reinforcement of a trial-and-error (try) coping strategy (Wong \& Amsel, 1976; Wong, 1977), and the differential in net incentive between the initial goal and alternative goals. Conflict resolution should be followed by a reduction in frustration and frustration-induced behavior. The dominance of goal-avoidance and substitution behavior in the resolution stage is therefore not so much due to the intensity of $\mathrm{r}_{\mathrm{F}}-\mathrm{S}_{\mathrm{F}}$ as due to the decision to abandon the initial goal. Previously, the intensity of $\mathrm{r}_{\mathrm{F}}-\mathrm{s}_{\mathrm{F}}$ was assumed to be a monotonic function of extinction trials (Amsel, 1967; Traupmann, Amsel, \& Wong, 1973). However, the findings of an inverted-U function of extinction-induced escape (Rosellini \& Seligman, 1975) and aggression (Thompson \& Bloom, 1966) are more compatible with the present conception of nonmonotonic function.

The stage model of frustration theory can be readily subjected to empirical test. The present series 
of experiments was designed to test several specific implications derived directly from the model.

\section{EXPERIMENTS 1 AND 2}

In an initial behavioral field analysis of partial reinforcement effects (Wong, 1977), the major finding was that continuous reinforcement (CRF) treatment led to greater response persistence than partial reinforcement (PRF), while PRF produced greater goal persistence than CRF. The present two experiments attempt to replicate this finding in two different strains of rats.

Because of insufficient extinction trials in Wong (1977), many subjects did not exhibit behavior characteristics of the resolution stage. In the present studies, extinction training was extended to test the following hypotheses derived from the stage model: (1) The frequency of response variation, hole exploration, and biting should be an inverted-U function of extinction trials. (2) CRF subjects are expected to have more practice in habitual routes, and hence more Stage 1 response persistence; they should therefore require more extinction trials to initiate response variation, hole exploration, and biting. (3) CRF subjects are expected to have less Stage 2 goal persistence and to reach the conflict resolution threshold faster because of greater frustration; they should be more inclined to engage in goal avoidance and substitution behavior.

\section{Method}

\section{Subjects}

The subjects were 20 male albino rats of Holtzman stock in Experiment 1 and 20 male hooded rats of Walker-Walker stock in Experiment 2. All subjects were bred in the vivarium of Trent University, and were approximately 90 days old at the beginning of the experimentation. They were housed in single cages with water always available, and maintained at $70 \%$ of free-feeding body weight during testing. Subjects in each strain were randomly divided into two groups of equal size.

\footnotetext{
Apparatus

The runway was made of pressboard and covered with a Plexiglas lid. The entire runway floor was made of metal grids. A frame, made of 1.2-cm-thick, 3-cm-high pressboard strips, was inserted in the alley section, dividing it into nine equal segments which were numerically coded to specify the route taken by each subject. An entrybox was attached to the startbox, separated by an aluminum door. Lifting the door disclosed a 10-cm-wide $x$ 14-cm-high opening, $5 \mathrm{~cm}$ above the entrybox floor. An escape box was attached to the goalbox, again separated by an aluminum door. Both entrybox emergence and escape from the goalbox involved jumping over a 5 - $\mathrm{cm}$-high hurdle. In conjunction with digital counters and clocks, photoelectric circuitry provided the following measures: latency to emerge from the entrybox into the startbox; time to traverse the first $50 \mathrm{~cm}$ (start measure), the second $50 \mathrm{~cm}$ (run), and the next $25 \mathrm{~cm}$ (goal); latency to escape into the escape box; frequencies of drinking and biting the model animals, and time in sand-digging. A more detailed description of the runway may be found in Wong (1977). The sand-digging assemblies installed in the startbox and goalbox were described in Wong, Roach, and Osborne (1975).
}

\section{Procedure}

Pretraining. Subjects were randomly matched and trained in pairs in all pretraining trials. They were first placed in the goalbox until each rat consumed about $2045-\mathrm{mg}$ Noyes pellets. They were then trained to go through swinging doors that were gradually lowered in three steps until they could push open free hanging doors. The foodcup was always baited with $2045-\mathrm{mg}$ pellets, to provide strong incentive for pushing through the swinging doors, but each rat was allowed only about $30 \mathrm{sec}$ acess to the foodcup. Two subjects in each experiment that failed to push through the two sets of swinging doors by the 5 th day of pretraining were discarded.

Acquisition. To start each trial, the subject was placed on the startbox platform which automatically activated the first clock. A maximum of $3 \mathrm{~min}$ was allowed for traversal of the alley and entry into the goalbox. Failing to complete the instrumental response within this time limit, the subject was gently guided into the goalbox and a score of $60.00 \mathrm{sec}$ was accorded to the uncompleted runway measures. On rewarded (R) trials, the subjects were allowed to consume four $45-\mathrm{mg}$ pellets and then confined in the goalbox for $30 \mathrm{sec}$. On nonreward $(\mathrm{N})$ trials, the subjects were confined in the unbaited goalbox for $30 \mathrm{sec}$.

PRF subjects were rewarded on $50 \%$ of the trials according to a predetermined quasi-random sequence with equiprobable $R-R$, R-N, N-N transitions.

All subjects were given 32 acquisition trials at two trials per day with an intertrial interval of approximately $20 \mathrm{~min}$.

Extinction. The subjects were placed in the entrybox. The lifting of the entrance door activated a clock which was stopped when the subject hurdle-jumped onto the startbox platform. If a subject failed to enter the goalbox in $3 \mathrm{~min}$, it was picked up and placed inside the goalbox. Following $30 \mathrm{sec}$ of confinement in the unbaited goalbox, the escape door was lifted and a clock was automatically activated. Once a subject had hurdlejumped into the escape cage, the escape door was closed and the clock was automatically stopped. If a subject failed to escape in $2 \mathrm{~min}$, it was guided into the escape cage.

In Experiment 1, the subjects received 40 extinction trials. In Experiment 2, because the experimenter had to be away, the subjects received only 32 extinction trials. All subjects were given two trials per day with an intertrial interval of approximately $30 \mathrm{~min}$.

The two experiments were conducted under identical conditions in close succession by the same experimenter.

\section{Results and Discussion}

\section{Acquisition}

Speed data. Time scores were converted to speeds (1/time). In Experiment 1, two subjects were discarded due to sickness and the results were based on eight subjects per group. Speed data for Experiments 1 and 2 are shown in Figures 2 and 3, respectively. PRF inferiority in the rate of acquisition was supported by a significant Reinforcement by Trial Blocks interaction in all three measures in Experiment 1, Fs $(7,476)=2.37,4.66,2.42$, respectively, $\mathrm{p}<.05$ in all cases. Similar interactions were obtained in Experiment 2; $\operatorname{Fs}(7,496)=2.57$, $4.62,7.86, p<.05$ in all cases.

Analysis of behavioral fields. Consistent with previous findings (Wong, 1977), PRF subjects showed more response variation. Mean number of different routes by PRF and CRF groups were 9.50 vs. 4.12, $\mathrm{t}(14)=5.33, \mathrm{p}<.01$ in Experiment 1 ; 8.55 vs. $4.88, \mathrm{t}(16)=5.87, \mathrm{p}<.01$ in Experiment 2 . 


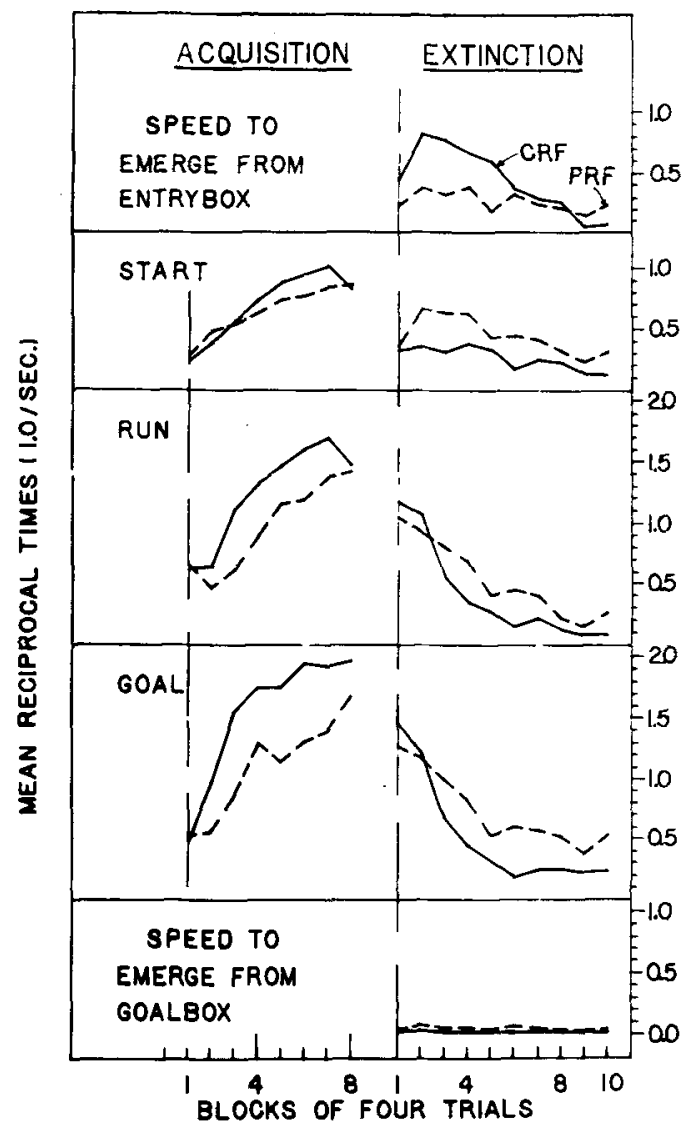

Figure 2. Speed data of Experiment 1 (Holtzman albino rats).

Frequency of head-poking into either one of the exploring boxes was used as an index of exploratory behavior. In Experiment 1, hole-exploration data were not recorded due to experimenter oversight. In Experiment 2, consistent with expectation, PRF subjects explored the hole more often, 2.22 vs. 0.44 , $\mathrm{t}(16)=2.67, \mathrm{p}<.05$.

The present findings of greater amount of route variation and hole exploration by PRF subjects were an extension of previous observations of more sniffing by rats (Wong, 1977) and more displacement pecking by doves (McFarland, 1966) under PRF conditions. McFarland (1966) concluded that his results supported Sutherland's $(1964,1966)$ view that nonreward switched attention and PRF subjects turned on more stimulus analyzers. According to Wong's (Note 1) analysis, a quasi-random partial reinforcement schedule necessarily involves uncertainty, conflict, and frustration, the conditions for evoking exploratory behavior (cf. Berlyne, 1966, 1967). It was also pointed out by Wong that exploration consists of an increase in the scope of response selection and stimulus selection, both of which should increase the likelihood of resolving conflict and removing the source of frustration. Therefore, the kinds of displacement activities observed in PRF conditions, far from being irrelevant, clearly serve an adaptive function.

Another important aspect of the behavioral iields was retracing, which consisted of recoiling and turning $180^{\circ}$ away from the goalbox after crossing the goaldoor threshold. Retracing could be considered as frustration-mediated avoidance (Amsel, 1967; Amsel, Rashotte, \& MacKinnon, 1966). PRF subjects retraced significantly more often than CRF subjects, 3.25 vs. $1.12, \mathrm{t}(14)=2.35, \mathrm{p}<.05$, in Experiment $1 ; 4.00$ vs. $1.44, t(16)=3.50, p<.01$, in Experiment 2. These data may be taken as evidence of the aversiveness of PRF conditions.

Drinking, biting, and sand-digging in the startbox occurred so rarely as to be nonexistent. Biting in the goalbox also rarely occurred. Sand-digging in the goalbox did not differ between CRF and PRF groups $(F<1)$, and showed a gradual decline during acquisition; the main effect of Trial Blocks was significant in Experiments 1 and 2, $F(7,98)=2.42, p<.05$, and $F(7,112)=22.90, p<.001$, respectively.

The inferior runway performance by PRF subjects could certainly be attributed to differences in incentive (cf. Logan, 1968), because they received a lower percentage of reinforcement, fewer reinforced trials, and a similar amount of overall rein-

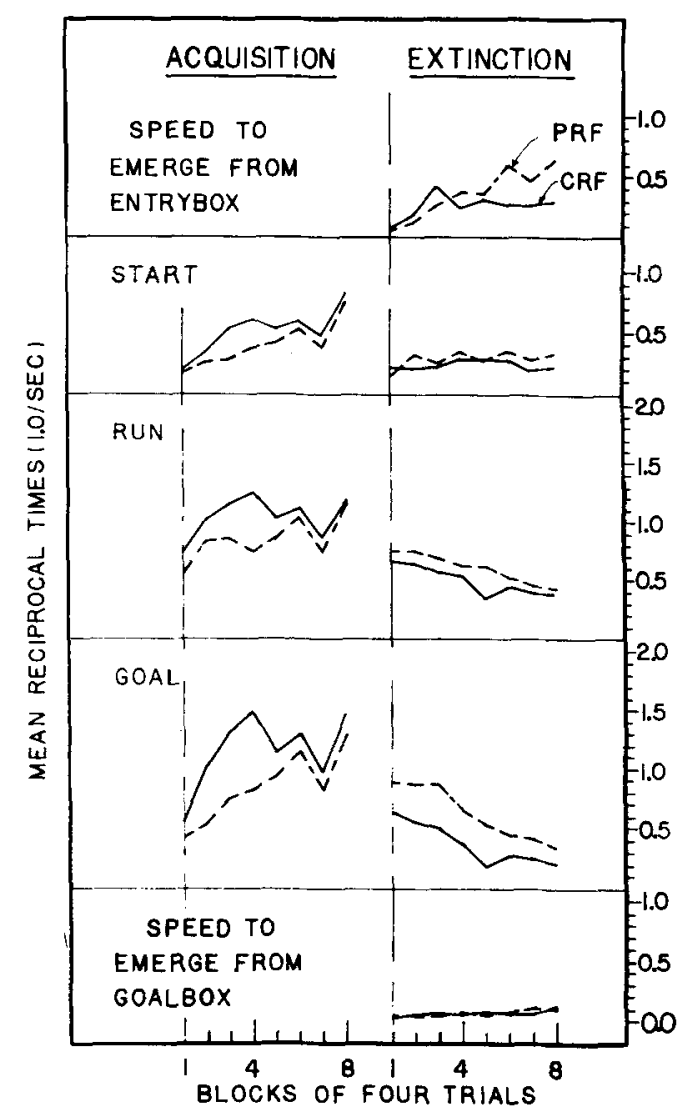

Figure 3. Speed data of Experiment 2 (Walker-Walker hooded rats). 
forcement. However, the analysis of behavioral fields has shown a higher level of PRF-induced investigatory behavior which should also interfere with speed performance. Even though the investigatory behavior was goal-directed, the fact that it involved exploring longer, unfamiliar routes, and various irrelevant aspects of the environment. should definitely increase the time in traversing the runway. It is also conceivable that the trial-anderror responding that characterized PRF subjects required longer latency in decision making, because on each trial the subject had to decide whether to try or not to try, and after choosing the former, it had to select a response from its response repertoire. Finally, the aversiveness of nonreward and the avoidance tendency should further reduce runway speed.

\section{Extinction}

Speed data. In both experiments, the conventional partial reinforcement extinction effect (PREE) is evident in Figures 2 and 3. In Experiment 1, the Reinforcement main effect was significant in the start measure, $F(1,14)=4.80, p<.05$, and the Reinforcement by Trial Blocks interaction was significant in the run and goal measures, $\operatorname{Fs}(9,546)$ $=3.15,3.10$, respectively, $\mathrm{p}<.01$ in both cases. Greater persistence of PRF subjects was also obtained in Experiment 2, where the Reinforcement main effect was significant in the goal measure, $F(1,16)=6.80, p<.05$, and the Reinforcement by Trial Blocks interaction was significant in the start, run, and goal measures, $\operatorname{Fs}(7,496)=3.25$, $11.66,22.57$, respectively, $\mathrm{p}<.01$ in all cases.

In both studies, CRF subjects emerged from the entrybox faster during the early stage of extinction, but PRF subjects emerged faster during the later stage, resulting in a significant Reinforcement by Trial Blocks interaction, $F(9,546)=2.70$ and $F(7,496)=2.73, p<.01$ in both cases. Since hurdlejumping into the startbox was never part of the previously reinforced response chain, this measure may be considered as an index of incentive motivation not confounded by the habit factor.

During the initial stage of extinction, CRF subjects should have greater incentive motivation, and therefore, faster hurdle-jumping. Further, greater anticipatory reward $\left(\mathrm{r}_{\mathrm{G}}-\mathrm{S}_{\mathrm{G}}\right)$ should result in greater primary frustration $\left(R_{F}\right)$. As anticipatory frustration $\left(r_{F}-S_{F}\right)$ eventually became conditioned to cues in the entrybox, CRF subjects should have less net incentive (cf. Logan, 1969), and hence slower hurdle-jumping later in extinction. This frustration-incentive interpretation was also applicable to the speed data in the start, run, and goal measures. However, additional processes must be invoked to account for qualitative changes in behavioral fields.

Analysis of behavioral fields. Since the behavioral fields induced by anticipatory frustration and primary frustration might be different, they are presented separately. Figure 4 shows major aspects of behavioral fields in pregoal segments of the runway.

The uppermost first panel shows that in both experiments, CRF subjects persisted longer in the habitual response topography, as supported by a significant difference in mean trials to disrupt a habitual goalpath on two consecutive trials, 12.87 vs. 4.44, $\mathrm{t}(14)=3.61, \mathrm{p}<.05$, in Experiment 1 , and 10.88 vs. $4.14, \mathrm{t}(16)=2.76, \mathrm{p}<.05$, in Experiment 2. Being more persistent in the habitual topographies, CRF subjects switched to alternative routes later, but discontinued the operant earlier than PRF subjects, as shown in the second panel and confirmed by a significant Reinforcement by Trial Blocks interaction in Experiment $1, F(9,126)$ $=1.96, \mathrm{p}=.05$. In Experiment 2, a similar pattern emerged, but the Reinforcement by Trial Blocks interaction was not significant. As predicted, a significant quadratic component was obtained in both experiments, $F(1,126)=43.59$ and $F(1,112)=$

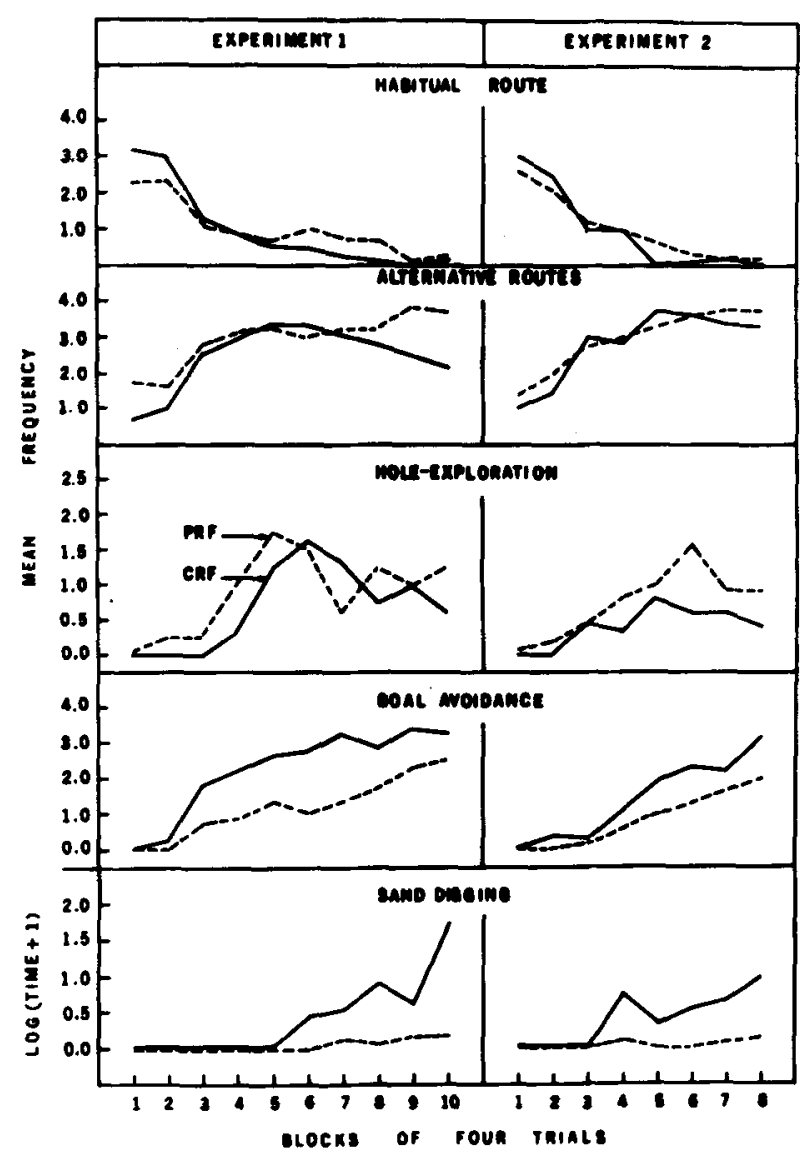

Figure 4. Behavioral fields in pregoalbox segments of the runway during extinction. For habitual routes, alternative routes, and goal avoidance, the maximum frequency was four because there were only four trials per block. For sand-digging, log transformation was based on total sand-digging lime per block. 
$31.39, \mathrm{p}<.001$ in both cases. Consistent with the stage model, CRF subjects were slower in initiating hole exploration. ${ }^{2}$ Mean trials to explore for CRF and PRF groups were 17.28 vs. $10.75, \mathrm{t}(14)=3.00$, $\mathrm{p}<.01$, in Experiment 1 ; and 14.4 vs. $10.3, \mathrm{t}(16)=$ $2.10, \mathrm{p}<.05$, in Experiment 2 . The prediction of an inverted-U function was again confirmed by a significant quadratic term in Experiments 1 and 2, $F(1,126)$ $=23.24$, and $F(1,112)=12.49$, respectively, $p<$ .001 in both cases.

The avoidance data in Figure 4 were based on retracing data and failure to enter the goalbox within the allotted time, the latter being a more stringent measure of Stage 2 goal persistence. It is quite apparent that PRF subjects had greater goal persistence, as evidenced by a significant main effect of Reinforcement in Experiments 1 and 2, $F(1,14)$ $=14.74$, and $F(1,16)=11.67$, respectively, $p<.01$ in both cases. Another indicator of Stage 2 persistence was the tendency to switch to subgoals or substitution behavior. In both experiments, CRF groups spent more time digging sand in the Startbox, $F(1,14)$ $=8.16$ and $F(1,16)=7.61, p<.05$ in both cases.

The analysis of behavioral fields has demonstrated that in extinction CRF and PRF groups predominated in different competing response tendencies and different persistence. As predicted, CRF subjects had a stronger tendency to avoid the goalbox and engage in substitution behavior (i.e., sand-digging), but showed greater response persistence; PRF subjects had a stronger tendency toward response variation and hole exploration, and showed greater goal persistence.

Greater response persistence by CRF subjects may be due to stronger habit strength. The positive correlations $(r=.65$ in Experiment $1, r=.69$ in Experiment 2, $\mathrm{p}<.01$ in both cases) between the amount of practice of the favored path and number of extinction trials to disrupt it provide some support for the habit hypothesis. Greater goal persistence by PRF subjects may be attributed to prior conditioning of a try strategy to cues of anticipatory frustration (Wong, 1977; Wong \& Amsel, 1976). This strategy-learning hypothesis is consistent with the finding that PRF subjects had a stronger tendency to engage in investigatory behavior during acquisition and extinction. In other words, they learned to try in the face of frustration.

Behavioral fields in the goalbox. Figure 5 depicts three major aspects of primary frustration reaction in the goalbox. In Experiment 1, biting increased over trials, $\mathrm{F}(9,126)=1.91, \mathrm{p}=.05$. Consistent with Wong (1977), PRF subjects had a slightly stronger tendency to bite towards the end of extinction, although neither the Reinforcement main effect nor the Reinforcement by Trial Blocks interaction was significant. In Experiment 2, the data were from only two subjects from each group; therefore, no statistical analysis was performed. In both experiments, the distribution of biting approximated an inverted-U function. Considering biting as a valid index of frustration reaction (e.g., Cherek \& Pickens, 1970), PRF subjects seemed more frustrated towards the end of extinction. This observation is in line with the stage model which predicts a reduction in frustration in the resolution stage. On the basis of goal avoidance and substitution behavior, CRF subjects did seem to reach the resolution stage earlier, and should therefore experience greater frustration reduction in the last stage of extinction.

Pertaining to extinction-induced goal escape (cf. Azrin, 1961; Rosellini \& Seligman, 1975), the model predicts an inverted-U function only in situations without the opportunity for an appetitive substitution behavior. In the present studies, the subjects did have a choice between escape and sand-digging; therefore, one cannot derive an unequivocal prediction from the model. Frequency of escape followed a pattern similar to speed of escape as shown in the last panel of Figures 2 and 3. PRF subjects seemed to have a slightly stronger tendency to escape. This escape behavior was reciprocally related to sanddigging in that, in both experiments, CRF subjects spent more time sand-digging, $F(1,14)=5.12$ and $F(1,16)=8.07, p<.05$ in both cases. It is likely that because CRF subjects switched more readily to sanddigging in the startbox, this tendency generalized to the goalbox.

Results of both experiments confirmed Wong's (1977) previous finding that CRF training increased response persistence, while PRF increased goal persistence. The prediction of an inverted-U function in investigatory and biting behavior received some empirical support. The kinds of competing responses which occurred in different stages of ex-

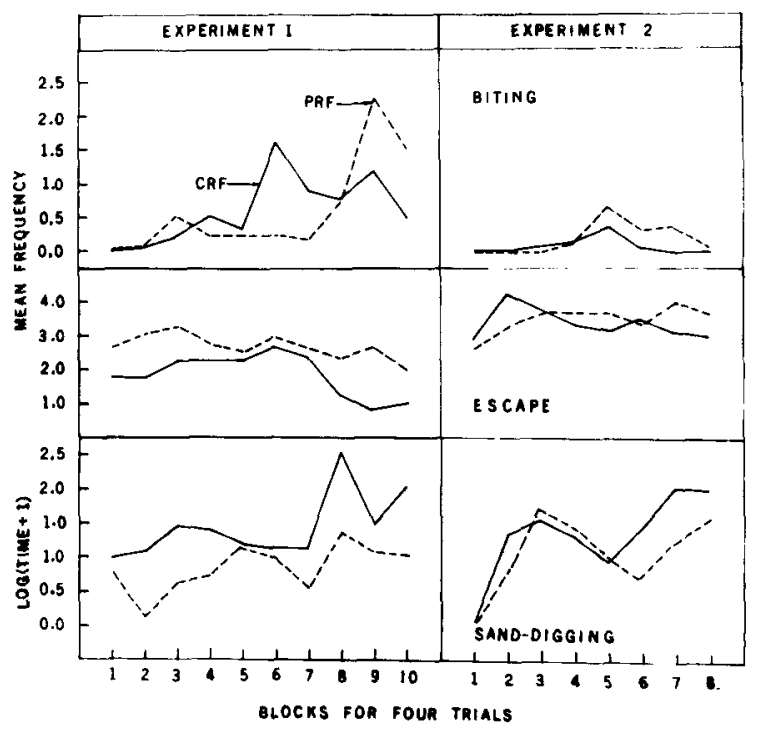

Figure 5. Behavioral fields in the goalbox during extinction. 
tinction also conformed to expectation. One may argue that in case of repeated failure it is only natural to first repeat the same response that has been successful (Stage 1), and then to try alternative responses (Stage 2) before switching to alternative goals (Stage 3). However, the model is more than a mere description of the obvious. The effects of various training parameters on different aspects of extinction behavior can be predicted on the basis of the model. The present two experiments have demonstrated the model's predictive power when percentage of reinforcement is varied. The next experiment was designed to test predictions related to other reinforcement parameters.

\section{EXPERIMENT 3}

Apart from percentage of reinforcement, the other two major training parameters that have been intensively investigated are magnitude of reinforcement and number of reinforced trials. In discretetrial runway studies, resistence to extinction tends to be inversely related to number of training trials and reward magnitude (e.g., Hulse, 1958; Ison, 1962; Ratliff \& Ratliff, 1971; Siegal \& Wagner, 1963; Tombaugh, 1967; Wagner, 1961). These findings have been considered an incentive phenomenon (Ison \& Cook, 1964; Theios \& Brelsford, 1964) and are readily derivable from Amsel's frustration theory. It has also been reported that training under lowdrive, small-reward conditions increased resistance to extinction (Traupmann, 1972), and this finding may be considered a habit phenomenon.

The above set of findings can be predicted from the stage model, even though the speed measure in the traditional narrow runway does not differentiate between response persistence and goal persistence. Given small reward and low drive, anticipatory frustration should grow so slowly that the $\mathrm{r}_{\mathrm{F}}-\mathrm{S}_{\mathrm{F}}$ intensity might not reach the resolution threshold in the usual number of extinction trials; therefore, runway speed could be largely determined by response persistence, which is known to be positively related to training trials (Wong, 1977; the present Experiments 1 and 2). Given high drive, the resolution stage is more likely to be reached, and this likelihood should be further increased with increase in reward magnitude and training trials because of greater $\mathrm{r}_{\mathrm{F}}-\mathrm{S}_{\mathrm{F}}$ intensity in extinction. Recall that the resolution stage is characterized by goal avoidance and substitution behavior, these competing responses should inevitably reduce runway speed. The traditional speed measure should, therefore, be inversely related to training trials and reward magnitude under high-drive conditions.

The present behavioral field approach permits separate assessment of response persistence and goal persistence, hence a more direct test of the above exposition. In the present study, training trials (60 vs. 21) and reward magnitude (500 mg vs. $97 \mathrm{mg}$ ) were orthogonally combined in high-drive subjects. It was predicted that subjects receiving 60 training trials should show greater response persistence, regardless of reward magnitude; large-reward subjects should show less goal persistence as reflected in retracing, substitution behavior, and the traditional speed measure. The least goal persistence was expected in the large-reward, long-training condition.

\section{Subjects}

\section{Method}

The subjects were 40 male hooded rats of the same origin as in Experiment 2 . They were maintained at $70 \%$ of free-feeding body weight throughout the experiment.

\section{Apparatus}

The apparatus was the same as that used in the two previous experiments, except for the following modifications. First, the frame used to partition the alleyway into nine segments for route identification was replaced by two parallel aluminum partitions $18 \mathrm{~cm}$ apart, forming three parallel alleyways of equal dimensions. These partitions were fastened to an aluminum floor placed directly on top of the original grid floor. Each aluminum partition had three $6 \times 6 \mathrm{~cm}$ openings, evenly spaced. Together, the nine openings were used to separate the original run section into 12 equal, but distinct, segments. This arrangement improved the accuracy of route identification. To increase the discriminability of the three parallel alleyways, the middle one was not painted, the left one was painted white, and the right one, black. The same colors were also applied to the respective startdoors and goal doors connected with the left and right alleyways. Second, the sand-digging assembly in the goalbox was sealed off by an aluminum sheet to permit a purer measure of goal escape latency during the extinction.

\section{Procedure}

The subjects were first trained to consume pellets in the goalbox in the same manner as in Experiments 1 and 2. This was immediately followed by acquisition training. The elaborate pretraining procedure employed in the previous two experiments was omitted, lest the treatment effects be masked by the many rewarded trials during pretraining. During acquisition, the subjects were given one trial on Day 1, two trials on Day 2, and three trials per day thereafter. The subjects receiving 60 trials (Group 60) began goalbox feeding and acquisition training first, while subjects receiving 21 trials (Group 21) were maintained on the same level of food deprivation and handled occasionally. Group 21 received their training just in time to complete the required acquisition trials on the same day as Group 60 . The subjects were gently guided into the goalbox when they failed to enter it within the allotted time. Six rats were discarded for failure to push through the doors by Day 3. Two others were discarded to equate ns across groups.

Each training group was subdivided into large- and smallreward groups, On each trial, under the large-reward condition, subjects received one $500-\mathrm{mg}$ pellet; under the small-reward condition, one $97-\mathrm{mg}$ pellet. The subjects were allowed to consume the reward pellet before they were removed from the goalbox.

All subjects began extinction at the same time. They received three trials per day for 10 consecutive days, with the goalbox unbaited on all trials. Compulsory goal confinement on each trial was $30 \mathrm{sec}$, after which the escape door was lifted. If the subject failed to hurdle-jump into an adjacent escape cage in $120 \mathrm{sec}$, it was gently guided into the escape cage. 
In addition to automatic recording of drinking, sand-digging, and model biting, the experimenter also recorded observed instances of hole exploration, grooming, and biting. In Experiment 2 , the model animal was almost shredded, yet the frequency count on the digital counter was very low, because a microswitch was triggered only when biting involved a lateral displacement of the model. To yield a more complete account of biting behavior, observed instances of model biting were manually recorded whenever the counter failed to register. Instances of biting directed to other parts of the runway were also manually recorded by checking off a behavioral check list.

As a displacement activity in conflict situations (Hinde, 1970; Zeigler, 1964), grooming tends to increase during extinction (Miller \& Stevenson, 1936; Wong, 1977). The present stage model predicts that extinction-induced grooming should eventually decrease once the conflict threshold is reached. To test this prediction, instances of grooming before goal entry and grooming after goal entry were recorded separately in the present experiment, because the former is probably more closely related to approach-avoidance conflict.

\section{Acquisition}

\section{Results and Discussion}

Speed data. With respect to the effect of reward magnitude on acquisition speed, the results, as depicted in Figure 6, were far from clear. Under the 60-trial condition, the small-reward group appeared slightly superior, but neither the main effect of Reward Magnitude nor any interaction involving Reward Magnitude was significant. Under the 21trial condition, the difference was more in line with traditional findings (e.g., Crespi, 1952; Logan, 1960; Wagner, 1961), as evidenced by a significant Reward Magnitude by Training Trials interaction in the start and goal measures, Fs $(6,280)=4.45$ and $4.60, p<$ .001 in both cases. Analysis of variance of the last two trial blocks based on all four groups failed to yield a significant main effect of Training Trials or Reward Magnitude. The present inconsistent findings were rather typical of the recent status of research on reward magnitude (cf. Mackintosh, 1974). The weight of recent evidence seems to indicate that contrary to earlier findings, reward magnitude does not have an unequivocal effect on either acquisition rate or acquisition asymptote.

Analysis of behavioral fields. None of the effects involving Reward Magnitude were significant in the analysis of various aspects of behavioral fields during acquisition. Consistent with previous findings (Wong, 1977), the acquisition process involved the narrowing of behavioral fields. All the collateral activities, (e.g., drinking, sand-digging) occurred almost exclusively in the first seven blocks of trials. Analysis of variance of data from the first seven trial blocks of Group 60 together with the entire seven trial blocks of Group 21 revealed only a significant main effect of Training Trials in favor of Group 60 in drinking, sand-digging, hole exploration, and number of different response routes, $F s(1,28)=$ $12.52,16.77,4.50,57.09, \mathrm{p}<.05$ in all cases. These differences could be due to the fact that Group 21 had been on food deprivation longer prior to acquisition training. The implication that the longer the food deprivation, the narrower the behavioral field remains to be tested directly.

\section{Extinction}

Speed data. In start, run, and goal measures, the traditional findings of faster rate of extinction by large-reward subjects can be detected in Figure 6. However, the Reward Magnitude by Trial Blocks interaction was significant only in the goal measure, $F(9,802)=5.18, p<.001$. Consistent with expectation, the graph also shows that training trials were negatively related to resistance to extinction only under larger reward conditions, resulting in a significant Training Trials by Reward Magnitude interaction in the start measure, $F(1,28)=4.57, p<.05$. The present results of speed data supported frustration theory, and confirmed the assumption that under high-drive conditions, the speed measure was more sensitive to incentive-frustration than to habit factors.

In entrybox emergence, Group 60 had a slower hurdle-jumping speed, $F(1,28)=11.9, p<.001$, probably due to a greater disruptive effect following a change in daily testing routine. When the subjects were first placed in the novel entrybox instead of the startbox during extinction, they seemed disoriented, spending much time alternatively in exploring and "freezing." Overtrained subjects seemed to persist longer in these competing responses and often required guidance to hurdle-jump into the startbox. According to the incentive-frustration analysis, it was expected that large-reward subjects should

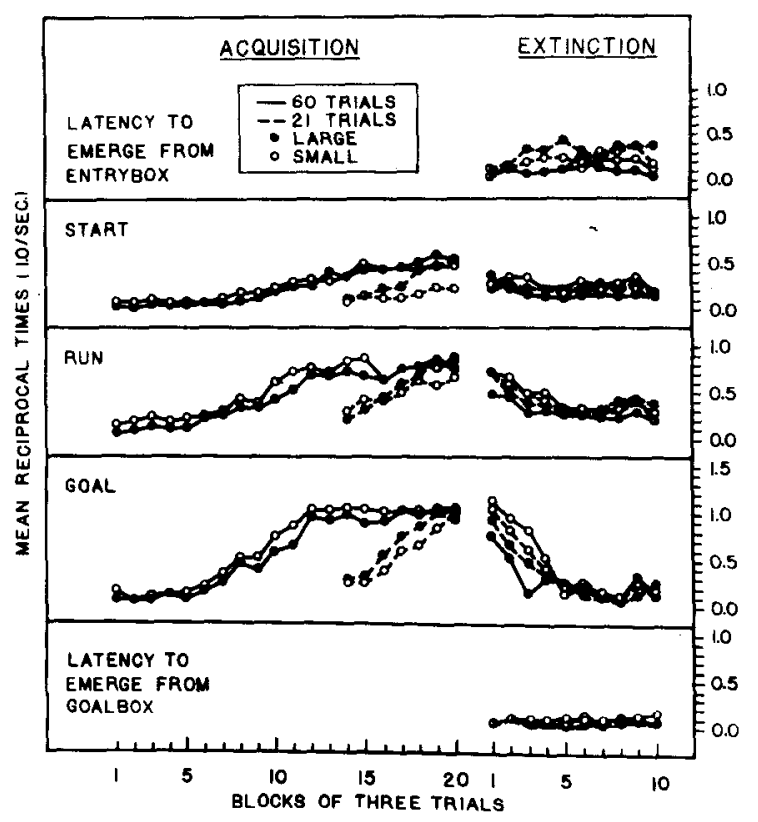

Figure 6. Speed data of Experiment 3. 
initially show faster hurdle-jumping speed because of greater incentive, but should be eventually inferior to small-reward subjects because of greater anticipatory frustration conditioned to entrybox cues and hence less net incentive. These predictions were only partially confirmed. Due to the strong disruptive effect previously described, both overtrained groups were equally slow in hurdle-jumping, but the eventual superior performance by the small-reward group conformed to prediction. For the 21 -trial subjects, the large-reward group showed initial superiority, as expected, but the predicted eventual crossover by the small-reward group was not realized within the given number of extinction trials. These present findings demonstrated that while entrybox emergence speed was sensitive to incentive-frustration variables, it was not completely free from confounding by the habit factor as previously assumed: overtraining seemed to have an adverse effect on this measure.

Experiments 1 and 2 showed that sand-digging was reciprocally related to the speed of escape from the goalbox. In the present experiment, without the opportunity of sand-digging in the goalbox, the escape measure should be a more reliable measure of the intensity of frustration. Figure 6 shows that small-reward subjects escaped faster, $F(1,28)=$ $5.0, p<.05$. This result seems to contradict frustration theory. However, the present stage model of frustration theory predicts a rapid decrease in frustration reaction once the conflict threshold is reached. The large-reward subjects, expected to encounter stronger frustration, should reach the conflict threshold faster and experience a reduction in frustration earlier. The model therefore predicted an initial superiority and a later inferiority in escape performance by large-reward subjects. This prediction was partially supported in that only the later inferiority was observed.

Analysis of behavioral fields. Various aspects of behavioral fields in the pregoal segments of the runway are depicted in Figure 7 . The uppermost panel shows that overtrained subjects persisted longer in habitual routes. Analysis of trials to disruption of habitual routes produced a significant main effect of Training Trials, $F(1,28)=13.90, p<.005$. Pearson's product-moment correlation between the frequency of occurrence of the habitual route during acquisition and trials to habit disruption was also significant, $r=0.68, p<.001$. Therefore, the prediction of $a$ positive relationship between response persistence and training trials was clearly substantiated. Other evidence of greater Stage 1 persistence by overtrained subjects was their slower initiation in hole exploration, $\mathrm{F}(1,28)=14.81, \mathrm{p}<.005$.

The stage model predicted an inverted- $U$ function for alternative routes, hole exploration, grooming, and biting various parts of the runway. Trend

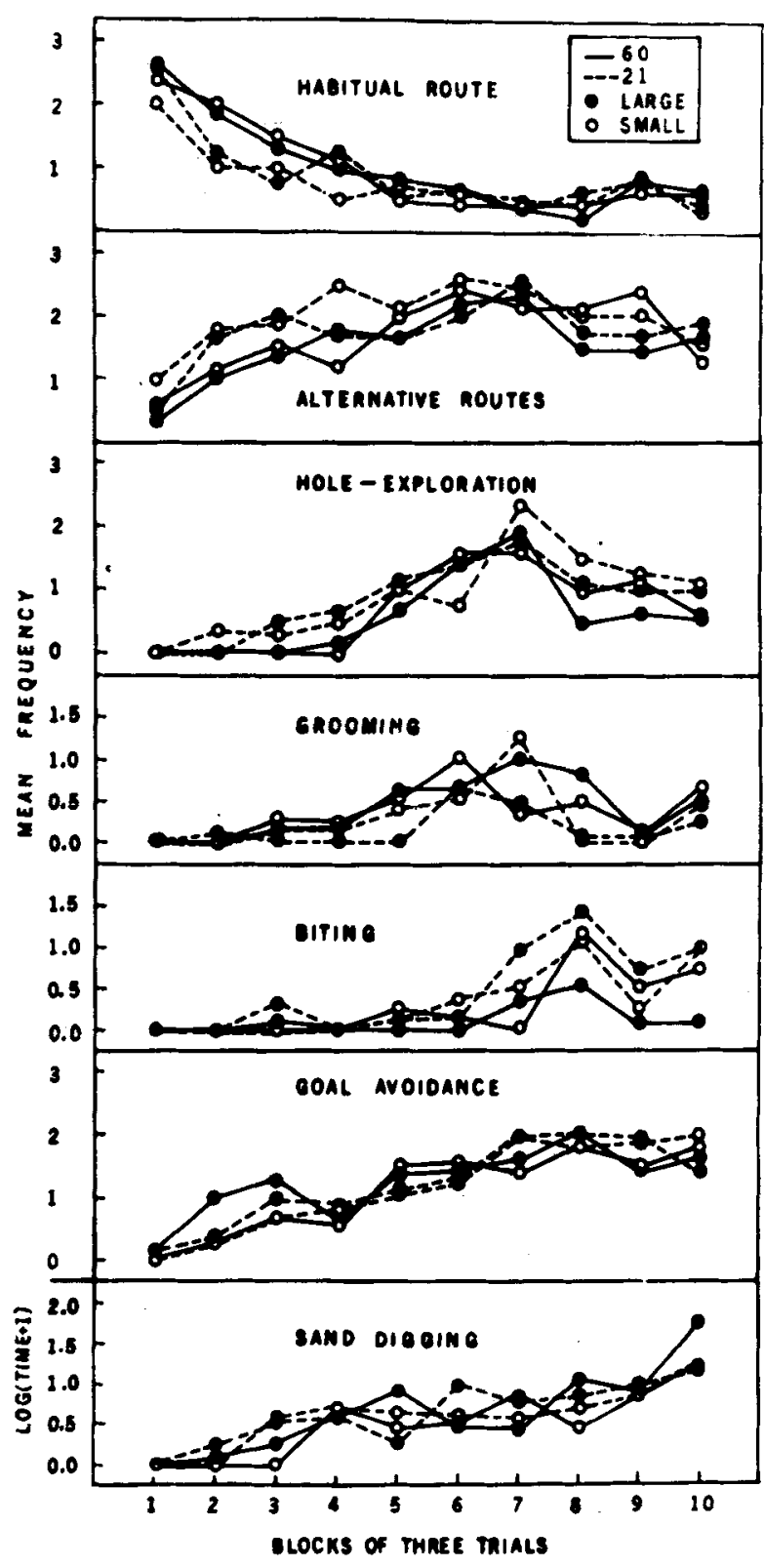

Figure 7. Behavioral fields in pregoalbox segments of the runway during extinction.

analysis revealed a significant quadratic component in the first three measures, $\operatorname{Fs}(1,252)=55.93,24.60$, and 5.92 , respectively, $p<.05$ in all three cases. Although Figure 8 does show an inverted-U function in biting, only the linear and cubic components were significant, $F(1,252)=54.67, p<.001, F(1,252)=$ $6.04, p<.05$, respectively. Consistent with the results of Experiments 1 and 2, drinking and model biting in the startbox occurred too infrequently for reliable analysis. Therefore, they were excluded from Figure 7.

Results pertaining to goal persistence were quite in line with predictions derived from the model. Figure 7 shows that large-reward groups exhibited goal 
avoidance earlier, as confirmed by a significant main effect of Reward Magnitude in trials to retrace, $\mathrm{F}(1,28)=5.13, \mathrm{p}<.05$. The model also predicted that large-reward groups should have a stronger tendency to switch to sand-digging, but this prediction was not borne out statistically.

Behavioral fields in the goalbox. Figure 8 shows an inverted-U function in grooming, biting various parts of the goalbox, and model biting. Trend analysis revealed a significant quadratic component in the first two measures only, $\operatorname{Fs}(1,252)=6.29$ and 15.18 , respectively, $\mathrm{p}<.05$ in both cases. In model biting, only the linear and cubic trends were significant, $F s(1,252)=53.20$ and 34.28 , respectively, $\mathrm{p}<.001$ in both cases; the absence of a significant quadratic trend might be due to the asymmetry of frequency distribution.

The eventual decrease in the frequency of goal escape, especially in the large-reward groups, provided further support for the concept of a resolution stage in extinction. The difference in escape frequency between two reward conditions paralleled that in escape speed, although only the latter was statistically significant. The stage interpretation previously accorded to the escape speed data was clearly applicable to the escape-frequency data.

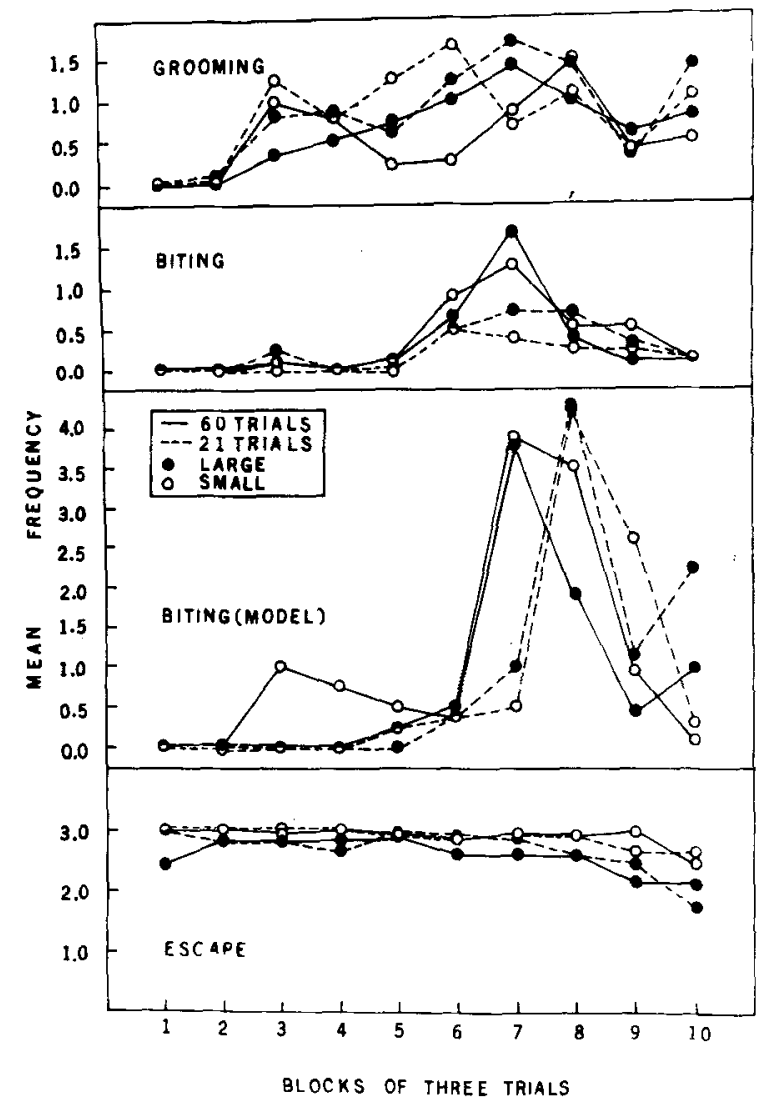

Figure 8. Behavioral fields in the goalbox during extinction.

\section{CONCLUDING DISCUSSION}

The results of the present experiments essentially agreed with the stage model of extinction. The major findings may be summarized as follows: (a) The initial stage of extinction was characterized by perseveration of habitual routes and the absence of competing responses. (b) The middle stage was characterized by an increase in investigatory behavior (i.e., route variation and hole exploration), biting behavior, and displacement grooming. (c) The last stage was characterized by a decrease in the above categories of competing responses but was dominated by goal avoidance and substitution behavior. (d) Response persistence was positively related to the amount of practice of habitual routes, implicating habit as an important determinant of transition from Stage 1 to Stage 2. (e) Goal persistence was increased by partial reinforcement (Experiments 1 and 2) and decreased by consistent large reward (Experiment 3), suggesting that the transition from Stage 2 to Stage 3 might be jointly modulated by the intensity of frustration encountered and the try strategy conditioned to frustrative cues.

It is difficult to conceive how the present results can be accounted for by any extinction theory that adopts a uniprocess view or excludes a competingresponse analysis. In the literature, experimental extinction in discrete-trial instrumental learning remains intractable. Thousands of white rats have run through the traditional simple runway, leaving as their legacy hundreds of experimental reports. Yet, we still do not have a comprehensive and adequate account of extinction in the simple runway! It may be mainly due to the problem that simplicity in experimental arrangements and measurements does not necessarily ensure simplicity in processes.

The present behavioral field approach has complicated the picture of extinction, and revealed some of the complexity of processes involved. The qualitative changes in behavioral fields in the course of extinction suggest a multiplicity of processes. From the perspective of frustration theory, extinction initially involves a decrease in anticipatory reward $\left(\mathrm{r}_{\mathrm{G}^{-}} \mathrm{s}_{\mathrm{G}}\right)$ and an increase in anticipatory frustration $\left(\mathrm{r}_{\mathrm{F}}-\mathrm{s}_{\mathrm{F}}\right)$, thus changing the internal stimulus component of the instigating stimulus complex. The decrement in the strength of habitual routes is then attributable to the process of stimulus generalization decrement. As the intensity $\mathrm{r}_{\mathrm{F}}-\mathrm{S}_{\mathrm{F}}$ increases, it begins to mediate competing responses, first investigatory behavior, then aggression, finally avoidance and substitution behavior, all of which necessarily interfere with goal approach speed. The transition from Stage 1 habitual mode of coping to Stage 2 trial-and-error mode of coping could be interpreted in terms of frustration-mediated competing re- 
sponses. The trial-and-error process in Stage 2 may be viewed as an active problem-solving process, such as that involved in Thorndike's puzzle box (cf. Thorndike, 1911). Therefore, theories of problemsolving should be applicable to this stage of extinction. Since Stage 2 also involves approach-avoidance conflict, various decision-making processes subsuming conflict behavior are implicated.

The transition from Stage 2 to Stage 3 is assumed to involve conflict resolution, conceptualized as a complex decision-making process. The resolution threshold may depend on both subjective probability of success and subjective criterion for quitting. Change in subjective probability may be affected by prior expectancy of success and the process of inhibitory learning (i.e., learning the absence of contingency between reinforcement and instrumental responding). Subjective criterion may also depend on a host of factors, such as past success in employing the try strategy, and an active process of evaluating the incentive differential between the initial goal and available alternative goals.

The foregoing account of extinction should help make the point that the extinction phenomenon is much more complex than hitherto assumed; that the different processes advocated by rival theoretical positions might all be involved at different stages of extinction. The new task that faces learning theorists is then to specify these processes as related to different stages. The present series of studies represented an initial step in this direction.

Finally, it is worth noting that one distinct advantage of the present behavioral field approach is that it provides a means of checking on the internal consistency of interpretation. To meet the requirement of internal consistency, the interpretation must be equally applicable to all aspects of behavioral fields. Therefore, any viable theory of extinction must simultaneously account for the quantitative change in the speed or probability of instrumental responding, and the qualitative changes in behavioral fields. The present stage model of frequency theory, however incomplete, has at least met the criterion of internal consistency.

\section{REFERENCE NOTE}

1. Wong, P. T. P. Exploratory behavior and rule learning. Presented in a memorial symposium in honor of the late Professor Daniel Berlyne at the Canadian Psychological Association, Vancouver, June 1977.

\section{REFERENCES}

AMSEL, A. The role of frustration nonreward in noncontinuous reward situations. Psychological Bulletin, 1958, 55, 102-119.

AMSEL. A. Partial reinforcement effects on vigor and persistence: Advances in frustration theory derived from a variety of withinsubjects experiments. In K. W. Spence \& J. T. Spence (Eds.), The psychology of learning and motivation (Vol. I)
Advances in research and theory. New York: Academic Press, 1967.

Amsel, A., Rashotte, M. E., \& Mackinnon, J. R. Partial reinforcement effects: Within-subject and between-subjects. Psychological Monograph, 1966, 80(20, Whole No. 628).

Amsel, A., Wong, P. T. P., \& Traupmann, K. L. Short-term and long-term factors in extinction and durable persistence. Journal of Experimental Psychology, 1971. 90. 90-95.

Antonitis, J. J. Response variability in the white rat during conditioning, extinction, and reconditioning. Journal of Experimental Psychology, 1951, 42, 273-281.

Azrin, N. H. Time-out from positive reinforcement. Science, $1961,133,382-383$.

Azrin, N. H., Hutchinson, R. R., \& Hake, D. F. Extinctioninduced aggression. Journal of the Experimental Analysis of Behavior, 1966, 9, 191-204.

BERLYNE, D. E. Curiosity and exploration: Animals spend much of their time seeking stimuli whose significance raises problems for psychology. Science, 1966, 153, 25-33.

BerLYNE, D. E. Arousal and reinforcement. Nebraska Symposium on Motrivation, 1967, 14, 1-110.

Bindra, D. The interrelated mechanisms of reinforcement and motivation, and the nature of their influence on response. Nebraska Symposium on Motivation, 1969, 17, 1-37.

Cherek, D. R., \& Pickens, R. Schedule-induced aggression as a function of fixed-ratio value. Journal of the Experimental Analysis of Behavior, 1970, 14. 309-312.

CRESPI, L. P. Quantitative variation of incentive and performance in the white rat. American Journal of Psychology, 1952, 55. 467:517.

FALK, J. L. The nature and determinants of adjunctive behavior. Physiology and Behavior, 1971, 6. 577-588.

Gleitman, H. Forgetting of long-term memories in animals. In W. K. Honig \& P. H. R. James (Eds.), Animal memory. New York: Academic Press, 1971.

GRAY, J. G. The psychology of fear and stress. New York: McGraw-Hill. 1971.

HINDE, R. A. Animal behavior: A synthesis of ethology and comparative psychology. New York: McGraw-Hill, 1970.

HulsE, S. H., JR. Amount and percentage of reinforcement and duration of goal confinement in conditioning and extinction. Journal of Experimental Psychology, 1958, 56, 48-57.

Ison, J. Experimental extinction as a function of number of reinforcements. Journal of Experimental Psychology, 1962, 64, 314-317.

Ison, J. R., \& Cook, P. E. Extinction performance as a function of incentive magnitude and number of acquisition trials. Psychonomic Science, 1964, 1, 245-246.

Jensen, G. D. Preference for bar pressing over "freeloading" as a function of number of rewarded presses. Journal of Experimental Psychology, 1963, 65, 451-454.

Kimble, G. A., \& Perlmuter, L. C. The problem of volition. Psychological Review, 1970, 77, 361-384.

KLINGER, E. Consequences of commitment to and disengagement from incentives. Psychologicl Review, 1975, 82, 1-25.

Klinger, E., Kemble, E. D., \& Barta, S. G. Cyclic activity changes during extinction in rats: A potential model of depression. Animal Learning \& Behavior, 1974, 2, 313-316.

Logan. F. A. Incentive. New Haven: Yale University Press, 1960.

LogAN, F. A. Incentive theory and changes in reward. In K. W. Spence \& J. T. Spence (Eds.), The psychology of learning and motivation (Vol. 2). New York: Academic Press, 1968. Pp. 1-30.

Logan, F. A. Punishment as negative incentive. In B. A. Campbell \& R. Church (Eds.), Punishment and aversive control. New York: Appleton-Century-Crofts, 1969. Pp. 43-54.

LogAN. F. A. Incentive theory, reinforcement and education. In R. Glaser (Ed.). The nature of reinforcement. New York: Academic Press, 1971. Pp. 45-61.

Mackintosh, N. J. The psychology of animal learning. London: Academic Press. 1974. 
MANDLER, G. The interruption of behavior. In D. Levine (Ed.), Nebraska Symposium on Motivation. 1964.

MARgulies, S. Response duration in operant level, regular reinforcement, and extinction. Joumal of Experimental Ana. lytical Behavior, 1961, 4, 317-322.

MCFARLAND, D. J. The role of attention in the disinhibition of displacement activities. Quarterly Journal of Experimental Psychology, 1966, 18, 19-30.

Miller, N. E. Liberalization of basic S-R concepts: Extensions to conflict behavior, motivation, and social learning. In S. Koch (Ed.), Psychology: A study of a science (Vol. 2). New York: McGraw.Hill, 1959. Pp. 196-293.

Miller, N. E., \& Stevenson, S. S. Agitated behavior of rats during experimental extinction and a curve of spontaneous recovery. Journal of Comparative Psychology, 1936, 21, 205-231.

Notterman, J. M.. \& Mintz, D. E. Dynamics of response. New York: Wiley, 1965.

RatlifF, R. G., \& Ratliff, A. R. Runway acquisition and extinction as a joint function of magnitude of reward and percentage of rewarded acquisition trials. Learming and Motivation, 1971, 2, 289-295.

Rosellini, R. A., \& Seligman, M. E. P. Frustration and learned helplessness. Journal of Experimental Psychology: Animal Behavior Processes, 1975, 1, 149-157.

SIEgel, S., \& W WGNER, A. R. Extended acquisition training and resistance to extinction. Journal of Experimental Psychology, 1963, 66, 3C,8-310.

Stolz. S. B.. \& Lotr, D. F. Establishment in rats of a persistence response producing a net loss of reinforcement. Journal of Comparative and Physiological Psychology, 1964, 57, 147-149.

Sutherland. N. S. The learning of discrimination by animals. Endeavor, 1964, 23, 140-152.

Sutherland, N. S. Partial reinforcement and breadth of learning. Journal of Experimental Psychology, 1966, 18, 289-302.

ThEIOs, J., \& BRELSFORD, J. Overlearning-extinction effect as an incentive phenomenon. Journal of Experimental Psychology, 1964. 67, 463-467.

Thompson, T., \& Bloom, W. Aggressive behavior and extinctioninduced response-rate increase. Psychonomic Science, 1966, 5, 335-336.

Thorndike. E. L. Animal intelligence: Experimental studies. New York: Macmillan, 1911.

Tомвaugh, T. N. The overtraining extinction effect with a discrete-trial bar-press procedure. Journal of Experimental Psychology, 1967, 73, 632-634.

Traupmann, K. L. Drive, reward, and training parameters, and the overlearning-extinction effect (OEE). Learning and Motivation. 1972, 3, 359-368.
Traupmann, K. L., Amsel, A., \& Wong, P. T. P. Persistence early and late in extinction as a function of number of continuous reinforcements preceding partial reinforcement training. Animal Learning \& Behavior, 1973, 1, 219-222.

WAGNER, A. R. Effects of amount and percentage of reinforcement and number of acquisition trials on conditioning and extinction. Journal of Experimental Psychology, 1961, 62 , 234.242.

WoNG, P. T. P. A behavioral field approach to instrumental learning in the rat: I. Partial reinforcement effects and sex differences. Animal Learning \& Behavior, 1977, 5, 5-13.

Wong, P. T. P., \& AMsel, A. Prior fixed ratio training and durable persistence in rats. Animal Learning \& Behavior. $1976,4,461-466$.

Wong, P. T. P., RoACh, T., \& OsBorne, B. A sand-digging apparatus for rats. Behavior Research Methods \& Instrumentation, 1975, 7, 34-36.

ZEIGLER, H. P. Displacement activity and motivational theory: A case study in the history of ethology. Psychological Bulletin, $1964,61,362-376$.

\section{NOTES}

1. Figure 5 shows a marked decrement for the CRF group in Block 4, and a decrement for both groups in Block 7. Due to circumstances beyond control, the experimenter took 5 days off prior to Block 4 and 3 days off prior to Block 7 . The decrement can be considered as evidence of retroactive interference (Gleitman, 1971). It seems that PRF subjects were less subject to retroactive interference. This view is consistent with the finding of greater spontaneous recovery and faster reacquisition following PRF training (Amsel, Wong, \& Traupmann, 1971).

2. Hole explorations after goal avoidance were not counted. Thus, the present hole exploration data can be considered as goal-directed investigatory behavior.

3. It should be noted that in both experiments, extinction began almost as soon as most PRF subjects began to fixate on a preferred route. PRF subjects may have more response persistence if they have the same amount of practice in habitual response topographies. However, this issue remains to be settled empirically.

(Received for publication May 3, 1977; revision accepted August 30, 1977.) 